\title{
Methods for the Explanation of Machine Learning Processes and Results for Non-Experts
}

\author{
Joachim Diederich \\ American University of Beirut \\ Department of Psychology \\ Riad El-Solh / Beirut 11072020 \\ Lebanon \\ jd43@aub.edu.lb
}

\section{Significance}

Machine learning systems such as artificial neural networks (ANNs) and support vector machines (SVMs) are commonplace in science, technology, business and health. However, the lack of an explanation capability is an impediment to the more wide-spread use of machine learning systems.

For more than 20 years, the field of rule extraction from ANNs and SVMs has tried to generate explanations for machine learning based decisions in the form of propositional, probabilistic or first-order rules (Diederich, 2008). At this point in time, however, there is no generally accepted technique that works for all machine learning systems. In addition, there is no method that is tailored to the needs of a user who may not be a domain expert and may not have a technology background. Ras et. al. (2018) classify naive users who are neither domain nor technical expert at various levels. Here, we assume that a user with limited technical and educational background would like to receive an explanation of a decision by a machine learning system.

While the algorithms and examples below focus on classifiers, in particular SVMs that learn binary decision tasks, the method can be generalised to machine learning systems that learn ranking function or regression, and even those that are based on rules in any format. Rules (e.g. in if-then-else format) do not guarantee human comprehensibility. New media is tailored for human understanding.

\section{Core idea}

The core idea here is to extract new media from machine learning systems to support human comprehensibility. Explanations are provided in the form of movie clips, audio files, graphic illustrations, text and other forms of multimedia. Explanations are based on storyboards that include examples from the data set that are important to decision making (e.g. support vectors, examples at the centre of important regions in decision space). Prior to including samples in storyboards, examples are transformed (simplified, enhanced or even replaced) to increase comprehensibility.

Explanations are tailored to the information need of individual users. While there are storyboard templates to support various types of explanation, storyboards are dynamic structures that change based on user feedback. Rules are generated as an in-between step or as an additional form of explanation. User acceptance is tested by use of parameters such as frequency of use of the explanation system, engagement with an explanatory dialogue and explicit forms of user feedback. 


\section{Background}

There are several types of explanation, e.g. causal explanations that are acceptable answers to "why" questions as opposed to the step-wise explanations that are acceptable responses to "how" questions. It is also possible to ask for clarifications if certain facts are known already and more detail is required. Finally, there are those types of questions that are best answered by providing an example for an event or fact. This example should be typical and therefore helps to explain a whole set of observations.

Thagard and Litt (2008) distinguish between three major explanatory processes:

- providing an explanation from available information,

- generating new hypotheses that provide explanations,

- and evaluating competing explanations.

Traditionally, the four major approaches for the generation of explanations are: (1) deductive, using logic or rule-based systems, (2) schematic, using explanation patterns or analogies; (3) probabilistic, using Bayesian networks; and (4) neural, using networks of artificial neurons (Thagard and Litt, 2008, p.2).

\section{Method}

The approach here is to generate storyboards as the basis for movie clips that explain the decisions of a machine learning system. Storyboards include samples from the data set, however, prior to inclusion in the storyboard samples are transformed (they are simplified by removing features, changed by including new features and their values, or samples are replaced by more typical examples). Samples that support a decision boundary (e.g. support vectors) or samples that are typical for a decision region may be included in storyboards.

There are several types of storyboards to allow different types of explanation: (1) Either to answer different types of user questions (How or Why) or (2) to provide different forms of explanation.

If the learning result of the underlying machine learning system is poor, e.g. due to noise or lack of data, explanations will be poor as well. It is then necessary to update the classifier. The explanation method outlined here allows the exploration of regions in decision space where generalisations do not hold. The user can then search for new data or select a different learning system to improve performance. Hence, algorithms that generate new hypotheses to explain data are provided as well.

\section{$\underline{4.1 \text { Algorithms }}$}

Here some brief descriptions of algorithms that are part of the method. All algorithms below assume that features have some form of meaning in isolation. That is, the method in its current form would not work for pixel-based representations as part of image processing. Future research will focus on feature representations that support explanation. Furthermore, different forms of explanation require different algorithms.

\subsubsection{Why questions}

There are two types of "Why" questions: (1) "Why did you ask this question" as part of an 
explanatory dialogue (a question posed by the system requires a clarification), and (2) "Why was this sample classified as X". The latter question can be answered by offering a similar, more typical example or by a storyboard that includes samples from both sides of the decision boundary.

In response to a "why was this example classified as $X$ " question for a decision made by an SVM that has learned a binary decisions task, the method will generate explanations in the following way:

Algorithm 1

1. Use decompositional methods for rule extraction from SVMs to determine regions (e.g. hyper-rectangles) on both sides of the decision boundary (e.g. by use of Nunez et al., 2002).

2. Identify the centres of the regions.

3. Identify the point in decision space that represents the sample that

triggered the "why question" (now called question sample, QS).

4. Retrieve a sample at the centre of the region that includes es (now called CS ) .

4.1 In a short version of this algorithm, simply replay CS with a statement for the user ("QS is similar to CS").

5. If CS is in multimedia format and of limited length:

5.1 Generate a storyboard or select a storyboard template.

5.2 Insert an introduction at position 1 in the storyboard ("QS was classified as $X$ because it is similar to CS and CS is typical for $\left.X^{\prime \prime}\right)$.

5.3 Use storyboard to generate movie clip.

5.4 Play movie clip.

6. If CS is in textual and/or numeric format and if the corresponding vector is high-dimensional (and possibly sparse),

6.1 Extract the most important features from CS (by use of the alphas or sensitivity analysis).

6.2 Generate a storyboard or select a storyboard template.

6.3 Insert an introduction at position 1 in the storyboard ("The following rule will explain why QS was classified as $X^{\prime \prime}$ ).

6.4 Form rule (by use of methods such as Nunez et al., 2002):

IF feature_1=value 1 and feature $2=$ value $2 \ldots$ feature $n=$ value $n$ THEN X.

6.5 Insert rule at position 2 in story board.

6.6 Use storyboard to generate movie clip.

6.7 Play movie clip.

Algorithm 2 provides a more detailed explanation why QS has been classified as X:

Algorithm 2

Go through steps $1-4$ of algorithm 1, disregard 4.1

5. If CS is in multimedia format and of limited length:

5.1 Cluster samples surrounding CS.

5.2 Select $n$ samples from the cluster.

5.3 Generate a storyboard.

5.4 Insert an introduction at position 1 in the storyboard ("QS was classified as $X$ because it is similar to these examples in class X").

5.5 Insert selected samples from the cluster at position 2 to $n$ in

the storyboard.

5.6 Use storyboard to generate movie clip.

5.7 Play movie clip.

Algorithms 1 and 2 can be generalised: Templates for storyboards are used to provide certain types of explanations. See the examples below. 


\subsubsection{How questions}

In response to a "how question", for decisions made by an SVM that has learned a binary decision task ("How does this automated system make decision?") and samples in multimedia format (audio/video):

Algorithm 3

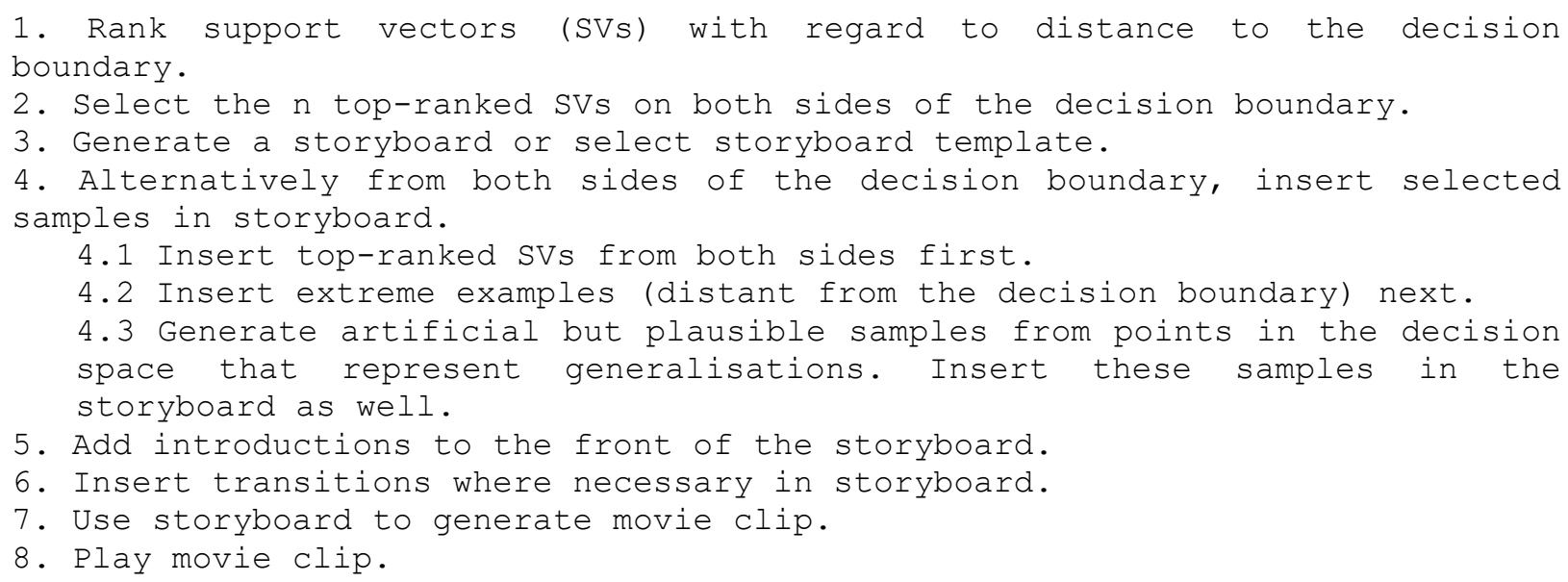

Instead of generating storybooks from scratch, storybook templates that represent certain types of explanations can be used. Samples in original and transformed format are then inserted into storyboards.

\subsubsection{Generate new hypotheses that explain data}

Decision boundaries can be moved and new ones can be created to explore new hypotheses. Schoelkopf et al. (1999) apply an SVM methodology to one-class classification problems. For instance, the origin can be treated as the only member of the second class. The decision boundary is then moved away from the origin and standard two-class SVM techniques are employed. Schoelkopf et al. (1999) describe the technique as follows: Suppose that a data set has a probability distribution $P$ in the feature space. Find a "simple" subset $S$ of the feature space such that the probability that a test point from $P$ lies outside $S$ is bounded by some a priori specified value (in Manevitz \& Yousef, 2001, p.142).

\section{Algorithm 4}

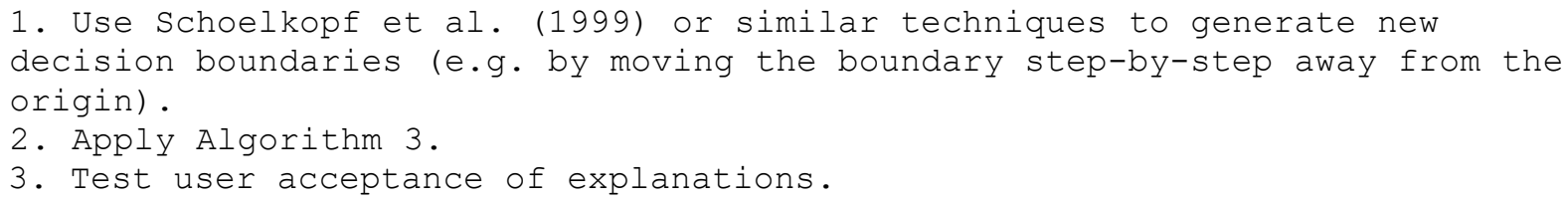

\subsubsection{Select the best features for explanation}

If QS and other samples are in mixed format (textual, numeric) and feature names are highly technical (abbreviations etc). 


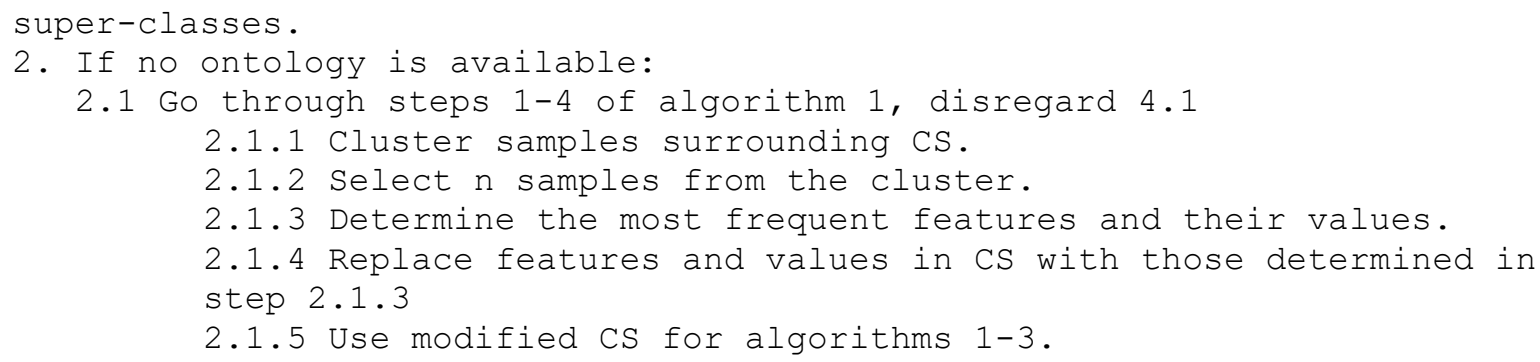

4.1.5 Create storyboards that demonstrate the difference between positive and negative examples

A storyboard can be based on a single example close to the decision boundary that changes its features while it slides over the decision boundary:

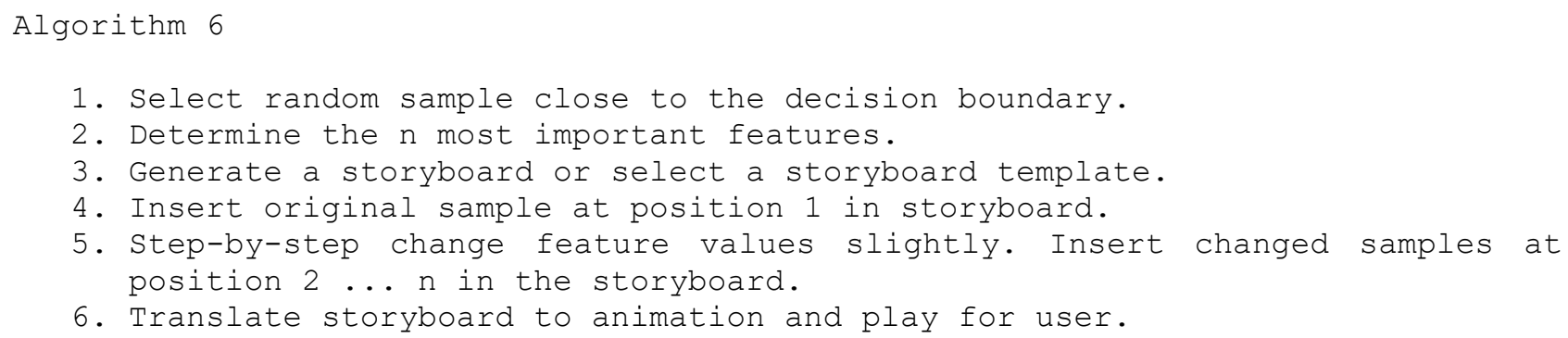

\subsubsection{Modify storyboards based on user experience}

Storyboards can be changed and adapted to individuals or groups of users by any learning algorithm for structural learning or time-series analysis (e.g. recurrent neural networks).

\section{Forms of Explanation: Examples}

\section{Explanation as Psychological Intervention}

Assume the underlying machine learning system has learned a binary decision task in the medical domain: cancer or not. A storyboard is used that includes samples that point to treatment options, recovery times and probabilities to return to work. The storyboard is designed so that it (1) correctly represents the decision of the machine learning system, and (2) provides an explanation that supports psychological recovery.

\section{Explanation for Education}

Assume the underlying machine learning system has learned to classify drawings of children into classes that correspond to a grading system. A student asks why s/he has received a poor mark. A storyboard is used that includes samples (drawings) from other students with similar grades, samples that resulted in better grades and samples that are right at the decision boundary. In addition, features that are relevant for classification are highlighted in samples that are part of the storyboard.

\section{References}

R. Andrews, R. Cable, J. Diederich, S. Geva, M. Golea, R. Hayward, C. Ho-Stuart, A.B. Tickle, An evaluation and comparison of techniques for extracting and refining rules from artificial neural networks._QUT NRC (February 1996). 
J. Diederich (Ed.), Rule Extraction from Support Vector Machines. Series: Studies in Computational Intelligence, Vol. 80. Berlin, Heidelberg, New York: Springer Verlag, 2008. ISBN: 978-3-540-75389-6.

L. Manevitz, M. Yousef. One-Class SVMs for Document Classification. Journal of Machine Learning Research 2 (2001) 139-154.

H. Núñez, C. Angulo, A. Catala. Rule extraction from support vector machines. In: ECAI-92 Proceedings of European Symposium on Artificial Neural Networks, 107-112, 2002.

G. Ras, M. van Gerven, P. Haselager, Explanation Methods in Deep Learning: Users, Values, Concerns and Challenges. To appear in Explainable and Interpretable Models in Computer Vision and Machine Learning, a Springer series on Challenges in Machine Learning, 2018.

B. Schoelkopf, J.C. Platt, J.Shawe-Taylor, A.J. Smola, and R.C. Williamson. Estimating the support of a high-dimensional distribution. Technical report, Microsoft Research, MSR-TR-99-87, 1999.

P. Thagard, A. Litt. Models of scientific explanation. In R. Sun (ed.), The Cambridge handbook of computational cognitive modeling. Cambridge: Cambridge University Press, 2008. 